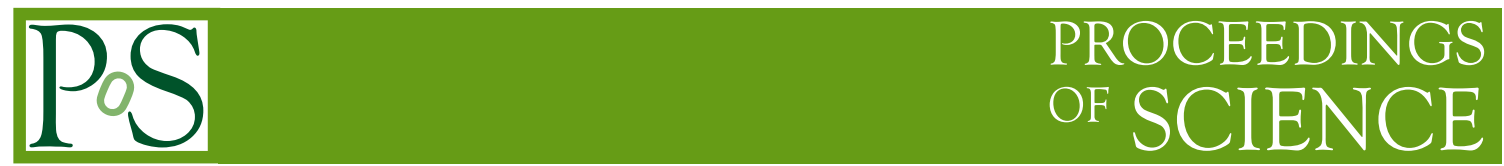

\title{
Hawking Fluxes and Covariant Anomalies
}

\section{Shailesh Kulkarni*}

S. N. Bose National Center for Basic Sciences, Block JD, sector- III, Salt lake, Kolkata 700098, India.

E-mail: shailesh@bose.res.in

Here we discuss in some detail two important approaches - the effective action approach given in [1] and the covariant anomaly mechanism [2] - to compute the Hawking radiation from generic spherically symmetric black hole. Further, we apply the covariant anomaly method to obtain the higher spin fluxes of Hawking radiation.

Black Holes in General Relativity and String Theory August 24-30 2008

Veli LoÅainj,Croatia

\footnotetext{
* Speaker.
} 


\section{Introduction}

Hawking radiation is one of the most important and prominent quantum effect in black hole physics. There exists a close resemblance between the black hole and laws of thermodynamics, even at the classical level [3]. However, Hawking [4] showed, by explicit computation of Bogoluobov coefficients between 'in' and 'out' state, that black holes do emit radiation and the spectrum of this radiation exactly matches with the thermal radiation coming from the black body kept at appropriate temperature. Apart from this there are several approaches $[5,6,7]$ to calculate the fluxes of Hawking radiation with each of them having their own merits and demerits. However, the fact that no one derivation is truly clinching has led to open problems leading to alternative approaches with fresh insights.

Anomaly might be recalled is a breaking of classical symmetry due to the process of quantization $[8,9]$. Specifically, for instance, a gauge anomaly is breakdown of gauge symmetry, taking the form of nonconservation of the gauge current. The cancellation gauge anomaly gives strong constraints on model building. Similarly, a gravitational anomaly is an anomaly in the general coordinate invariance and it is manifested in the nonconservation of energy-momentum tensor. Role of the anomaly in deriving the Hawking radiation is not surprising. Indeed, by using the trace anomaly Christensen and Fulling [10] were able to reproduce the expected results. Although this method is restricted for conformal fields propagating in $(1+1)$ dimensional black hole background, it nevertheless indicate the importance of the quantum anomaly in the context of Hawking radiation. Recently, Wilczek and collaborators [11, 12] gave an interesting method to compute the Hawking fluxes using chiral gauge and gravitational anomalies. Unlike the trace anomaly approach this method is expected to hold in any dimensions. However, an unpleasant feature of $[11,12]$ was that while the expressions for anomalies were taken to be consistent, the boundary condition required to fix the arbitrary constants were covariant. Hawking fluxes were then obtained by cancellation of the consistent anomalies. This was rectified by us [13] by providing a simplified derivation using only covariant expressions of anomalies as well as boundary condition.

The approaches of $[11,12,13]$ are based on the fact that effective field theory near the horizon become two dimensional. From this two dimensional theory if we formally remove the modes which are going in to the black hole then the effective theory becomes chiral. Any two dimensional chiral theory possesses gravitational, and if gauge fields were present, gauge anomalies. Such anomalous theories admit two types of anomalous currents and energy-momentum (EM) tensors: the consistent and the covariant $[8,9,14,15]$. The covariant divergences of these currents/EM tensors yields either consistent or covariant gauge/gravitational anomalies $[8,9,15,16,17]$. The consistent currents and anomaly satisfy the Wess-Zumino condition but do not transform covariantly under gauge or general coordinate transformation while the opposite is true for covariant currents and anomaly. Similar conclusions also hold for gravitational case.

In our approach [13] we have reformulated the analysis of [11,12] in terms of only covariant expressions. The consistent expressions were completely bypassed. There are two distinct advantages of such an approach. First, since the covariant boundary conditions are necessary, it is conceptually clean to discuss whole analysis from covariant point of view. Another point is that the calculations become much simpler and compact. Also, as we shall discuss later, the covariant anomaly approach is much suitable to compute the higher spin fluxes of Hawking radiation. In 
this case it is essential to use the covariant expressions since, only the covariant expressions of anomalies and higher spin current are known [18].

In another new development, also based on the chiral gauge and diffeomorphism anomaly, Hawking radiation was obtained by us [1]. An important ingredient in this approach was that, unlike the anomaly cancellation mechanism $[11,12,13]$, only the expressions of chiral covariant currents and covariant EM tensors, obtained from the near horizon chiral effective action, together with the covariant boundary condition, were shown to be sufficient to compute the Hawking fluxes. The starting point is the structures for the two dimensional effective actions which are known for the chiral (anomalous)[19] and usual (anomaly free) [20,21] cases. The relevant expressions for the covariant gauge current and the covariant EM tensor are obtained by taking appropriate functional derivatives of the chiral effective action. The arbitrary constants are fixed by imposing the covariant boundary condition namely, the vanishing of covariant current and the covariant EM tensor at the event horizon. The Hawking fluxes are then correctly reproduced by taking the asymptotic infinity limit of covariant currents and EM tensors. Finally, we note that the asymptotic forms for currents and EM tensors obtained from this analysis are exactly matches with the asymptotic values of currents and EM tensors computed form the usual effective action [20,21]. Note that all the above approaches [1, 2, 12, 13, 20], to compute the Hawking flux, uses the same boundary condition. Naturally, one would seek for further clarification of the covariant boundary condition. Indeed, in our very recent work [22], also based on chiral effective action approach [1], we provide a detailed explanation of this covariant boundary condition. There we noted that the current and EM tensor obtained by solving the anomaly equations subjected to the covariant boundary condition agrees exactly with the result derived from the chiral effective action but by imposing the boundary condition appropriate for the Unruh vacuum.

There is one more quick and efficient method to obtain the Hawking flux briefly introduced in [2] which uses only the knowledge of covariant anomalies near the horizon. Contrary to earlier approaches $[11,12,13,20]$ a splitting of the space in different regions (near to and away from the horizon) using discontinuous step functions were avoided. As we shall discuss in subsequent section, the computation of the Hawking fluxes associated with higher spin currents can be done easily in this approach.

The organization of this paper is as follows. The calculation of Hawking flux from generic spherically symmetric black hole using chiral effective action is discussed in section 2. Also, the role of chirality in imposing constraints on the structure of the current/EM tensor is elucidated. In section 3 we compute the higher spin fluxes the Hawking radiation. In particular, we adopt the covariant anomaly method [2] to compute the fluxes associated with the spin three current. Our concluding remarks are contained in section 4.

\section{Hawking fluxes from chiral effective action}

Consider a generic spherically symmetric black hole represented by the metric,

$$
d s^{2}=f(r) d t^{2}-\frac{1}{h(r)} d r^{2}-r^{2}\left(d \theta^{2}+\sin ^{2} \theta d \phi^{2}\right)
$$

where $f(r)$ and $h(r)$ are the metric coefficients. The event horizon is defined by $f\left(r_{h}\right)=h\left(r_{h}\right)=0$. Also, in the asymptotic limit the metric (2.1) become Minkowskian i.e $f(r \rightarrow \infty)=h(r \rightarrow \infty)=1$ 
and $f^{\prime \prime}(r \rightarrow \infty)=f^{\prime \prime \prime}(r \rightarrow \infty)=h^{\prime \prime}(r \rightarrow \infty)=h^{\prime \prime \prime}(r \rightarrow \infty)=0$.

Now consider quantum fields (scalar or fermionic)propagating on this background. It was shown that $[11,12]$, by using a dimensional reduction technique, the effective field theory near the event horizon becomes two dimensional with the metric given by the $r-t$ section of (2.1)

$$
d s^{2}=f(r) d t^{2}-\frac{1}{h(r)} d r^{2} .
$$

Note that $\sqrt{-g}=\sqrt{-\operatorname{detg}_{\mu \nu}}=\sqrt{\frac{f}{h}} \neq 1$ (unless $f(r)=h(r)$ ). On this two dimensional background, the modes which are going in to the black hole (for example left moving modes) are lost and the effective theory become chiral. The effective action representing such chiral theory is known in the literature [19]. We adopt the following strategy. The expressions for covariant current and the covariant EM tensor will be deduced from this chiral effective action, suitably modified by local counterterm. Local structures are obtained by introducing auxiliary variables whose solutions contain arbitrary constants. These constants are fixed by imposing appropriate boundary conditions.

The two dimensional chiral effective action $[1,19]$ is defined as,

$$
\Gamma_{(H)}=-\frac{1}{3} z(\omega)+z(A)
$$

where $A_{\mu}$ and $\omega_{\mu}$ are the gauge field and the spin connection, respectively, and,

$$
z(v)=\frac{1}{4 \pi} \int d^{2} x d^{2} y \varepsilon^{\mu v} \partial_{\mu} v_{v}(x) \Delta^{-1}(x, y) \partial_{\rho}\left[\left(\varepsilon^{\rho \sigma}+\sqrt{-g} g^{\rho \sigma}\right) v_{\sigma}(y)\right]
$$

Here $\Delta^{-1}$ is the inverse of d'Alembertian $\Delta=\nabla^{\mu} \nabla_{\mu}=\frac{1}{\sqrt{-g}} \partial_{\mu}\left(\sqrt{-g} g^{\mu v} \partial_{v}\right)$ and $\varepsilon^{t r}=-\varepsilon^{r t}=$ $-\varepsilon_{t r}=\varepsilon_{r t}=1$. From a variation of this effective action the energy momentum tensor and the gauge current are computed. These are shown in the literature $[8,9,14,15,16,17]$ as consistent forms. To get their covariant forms in which we are interested, however, appropriate local polynomials have to be added. This is possible since energy momentum tensors and currents are only defined modulo local polynomials. Thus we obtain,

$$
\delta \Gamma_{H}=\int d^{2} x \sqrt{-g}\left(\frac{1}{2} \delta g_{\mu v} T^{\mu v}+\delta A_{\mu} J^{\mu}\right)+l
$$

where the local polynomial is given by [19],

$$
l=\frac{1}{4 \pi} \int d^{2} x \varepsilon^{\mu v}\left(A_{\mu} \delta A_{v}-\frac{1}{3} w_{\mu} \delta w_{v}-\frac{1}{24} R e_{\mu}^{a} \delta e_{v}^{a}\right)
$$

The covariant energy momentum tensor $T^{\mu v}$ and the covariant gauge current $J^{\mu}$ are read-off from the above relations as [1, 19],

$$
\begin{array}{r}
T^{\mu}{ }_{v}=\frac{e^{2}}{4 \pi}\left(D^{\mu} B D_{v} B\right) \\
+\frac{1}{4 \pi}\left(\frac{1}{48} D^{\mu} G D_{v} G-\frac{1}{24} D^{\mu} D_{v} G+\frac{1}{24} \delta_{v}^{\mu} R\right)
\end{array}
$$




$$
J^{\mu}=-\frac{e^{2}}{2 \pi} D^{\mu} B
$$

Note the presence of the chiral covariant derivative $D_{\mu}$ expressed in terms of the usual covariant derivative $\nabla_{\mu}$,

$$
D_{\mu}=\nabla_{\mu}-\bar{\varepsilon}_{\mu v} \nabla^{v}=-\bar{\varepsilon}_{\mu v} D^{v}
$$

where $\bar{\varepsilon}_{\mu v}=\sqrt{-g} \varepsilon_{\mu v}$ and $\bar{\varepsilon}^{\mu v}=\frac{1}{\sqrt{-g}} \varepsilon^{\mu v}$. The auxiliary fields $B$ and $G$ in $(2.7,2.8)$ are defined as

$$
\begin{aligned}
B(x) & =\int d^{2} y \sqrt{-g} \Delta^{-1}(x, y) \bar{\varepsilon}^{\mu v} \partial_{\mu} A_{v}(y) \\
G(x) & =\int d^{2} y \sqrt{-g} \Delta^{-1}(x, y) R(y)
\end{aligned}
$$

so that they satisfy

$$
\begin{aligned}
\Delta B(x) & =\bar{\varepsilon}^{\mu v} \partial_{\mu} A_{v}(x) \\
\Delta G(x) & =R(x)
\end{aligned}
$$

where $R$ is the two dimensional curvature scalar, and for the metric (2.2) it is given by

$$
R=\frac{f^{\prime \prime} h}{f}+\frac{f^{\prime} h^{\prime}}{2 f}-\frac{f^{\prime 2} h}{2 f^{2}}
$$

It is possible to reproduce the covariant gravitational/gauge Ward identities $[1,8,9,16,17,19]$ from (2.7,2.8). For example, using (2.8), (2.10) and (2.12), we find,

$$
\nabla_{\mu} J^{\mu}=-\frac{e^{2}}{2 \pi} \Delta B=-\frac{e^{2}}{2 \pi} \bar{\varepsilon}^{\mu v} \partial_{\mu} A_{v}=\frac{-e^{2}}{4 \pi \sqrt{-g}} \varepsilon^{\mu v} F_{\mu v}
$$

This is the expression for covariant gauge anomaly $[8,9]$. Similarly, we can easily show, by using (2.7-2.13), that the EM tensor (2.7) satisfy the covariant gravitational Ward identity, given by [1, 19],

$$
\nabla_{\mu} T^{\mu v}=J_{\mu} F^{\mu v}+\frac{1}{96 \pi} \bar{\varepsilon}^{v \mu} \nabla_{\mu} R
$$

The first term in the above expressions is the classical Lorentz force term, while the second term is the covariant gravitational anomaly $[16,17]$. Note also the presence of the covariant trace anomaly following from (2.7)

$$
T_{\mu}^{\mu}=\frac{R}{48 \pi} \text {. }
$$

The chiral theory has both a diffeomorphism anomaly and a trace anomaly (2.17). This is distinct from the vector case where there is only a trace anomaly $T_{\mu}^{\mu}=\frac{R}{24 \pi}$ while the diffeomorphism invariance kept intact.

Solutions of (2.12) and (2.13) are given by,

$$
B(x)=B_{o}(r)-a t+b ; \partial_{r} B_{o}=\frac{A_{t}(r)+c}{\sqrt{f h}}
$$

and

$$
G=G_{o}(r)-4 p t+q ; \partial_{r} G_{o}=-\frac{1}{\sqrt{f h}}\left(\frac{f^{\prime}}{\sqrt{-g}}+z\right)
$$


where $a, b, c, p, q$ and $z$ are constants. Now, by substituting (2.18) in (2.8) we obtain,

$$
\begin{aligned}
& J^{r}(r)=\frac{e^{2}}{2 \pi \sqrt{-g}}\left[A_{t}(r)+c+a\right] \\
& J^{t}(r)=\frac{e^{2}}{2 \pi f}\left[A_{t}(r)+c+a\right]=\frac{\sqrt{-g}}{f} J^{r} .
\end{aligned}
$$

Likewise, by using $(2.18,2.19)$ in $(2.7)$ we find

$$
\begin{aligned}
T_{t}^{r} & =\frac{e^{2}}{4 \pi \sqrt{-g}} \bar{A}_{t}^{2}(r)+\frac{1}{12 \pi \sqrt{-g}} \bar{P}^{2}(r)+\frac{1}{24 \pi \sqrt{-g}}\left[\frac{f^{\prime}}{\sqrt{-g}} \bar{P}(r)+\bar{Q}(r)\right] \\
T_{r}^{r} & =\frac{R}{96 \pi}-\frac{\sqrt{-g}}{f} T^{r}{ }_{t} \\
T^{t}{ }_{t} & =-T^{r}{ }_{r}+\frac{R}{48 \pi}
\end{aligned}
$$

with $\bar{A}_{t}(r), \bar{P}(r)$ and $\bar{Q}(r)$ defined as

$$
\begin{aligned}
\bar{A}_{t}(r) & =A_{t}(r)+c+a \\
\bar{P}(r) & =p-\frac{1}{4}\left(\frac{f^{\prime}}{\sqrt{-g}}+z\right) \\
\bar{Q}(r) & =\frac{1}{4} h f^{\prime \prime}-\frac{f^{\prime}}{8}\left(\frac{h f^{\prime}}{f}-h^{\prime}\right) .
\end{aligned}
$$

From equations $(2.20,2.21)$ it is evident that there is only one independent component of $J^{\mu}$. The point is that current $J^{\mu}(2.8)$, derived from the chiral effective action, satisfy the chirality condition [22]

$$
J_{\mu}=-\bar{\varepsilon}_{\mu v} J^{v},
$$

which fixes one of the component of $J^{\mu}$. This can be further illustrated by transforming $J^{\mu}$ to null coordinates given by

$$
\begin{aligned}
& v=t+r * ; \frac{d r}{d r *}=\sqrt{f h} \\
& u=t-r *
\end{aligned}
$$

The metric (2.2) in these coordinates looks like

$$
d s^{2}=\frac{f(r)}{2}(d u d v+d v d u) .
$$

Then by substituting $\mu=v$ in the chirality condition (2.28) we get

$$
J_{v}=0
$$

while $J_{u}$ cannot be determined, solely, from the chirality criterion. Similar considerations also holds for stress tensor $T^{\mu v}(2.7){ }^{1}$.

\footnotetext{
${ }^{1}$ See [22] for detail discussion of chirality.
} 
Next, we fix the arbitrary constants appearing in the expression of current $J^{r}(2.20)$ and stress tensor $T^{r}{ }_{t}$ (2.22) by imposing the covariant boundary condition [1, 2, 12, 13, 20], namely, the vanishing of covariant current and covariant EM tensor at the horizon i.e

$$
\begin{aligned}
J^{r}\left(r=r_{h}\right) & =0 \\
T^{r}{ }_{t}\left(r=r_{h}\right) & =0,
\end{aligned}
$$

this yields, from (2.20)

$$
c+a=-A_{t}\left(r_{h}\right)
$$

while, from equation (2.22) we get

$$
p=\frac{1}{4}\left(z \pm \sqrt{f^{\prime}\left(r_{h}\right) h^{\prime}\left(r_{h}\right)}\right)
$$

Finally, by substituting (2.35) in (2.20) we obtain

$$
J^{r}(r)=\frac{e^{2}}{2 \pi \sqrt{-g}}\left[A_{t}(r)-A_{t}\left(r_{h}\right)\right]
$$

and the other component $J^{t}$ is determined from (2.21). Similarly, from (2.22) and $(2.35,2.36)$ we get the expression for $T_{t}^{r}$ :

$$
\sqrt{-g} T_{t}^{r}(r)=\frac{e^{2}}{4 \pi}\left[A_{t}(r)-A_{t}\left(r_{h}\right)\right]^{2}+\left[N_{t}^{r}(r)-N_{t}^{r}\left(r_{h}\right)\right]
$$

where,

$$
N_{t}^{r}=\frac{1}{96 \pi}\left(h f^{\prime \prime}+\frac{f^{\prime} h^{\prime}}{2}-\frac{f^{\prime 2} h}{f}\right)
$$

Now we observe that the covariant gauge (2.15) as well as gravitational (2.16) anomaly vanish at $(r \rightarrow \infty)$ limit. Thus, we can compute the Hawking charge and energy flux, which has to be measured at asymptotic infinity, by taking the asymptotic infinity limit of $J^{r}(r)(2.37)$ and $T^{r}{ }_{t}(r)$ (2.39).

$$
\begin{aligned}
& \text { Chargeflux }=J^{r}(r \rightarrow \infty)=-\frac{e^{2}}{2 \pi} A_{t}\left(r_{h}\right), \\
& \text { Energyflux }=T_{t}^{r}(r \rightarrow \infty)=\frac{e^{2}}{4 \pi} A_{t}^{2}\left(r_{h}\right)+\frac{1}{192 \pi} f^{\prime}\left(r_{h}\right) h^{\prime}\left(r_{h}\right) .
\end{aligned}
$$

These are the desired expressions of Hawking charge and energy flux from the generic spherically symmetric black hole and it agrees with the result obtained by anomaly cancellation approach [23].

\section{Higher spin fluxes via covariant anomaly}

In this section we compute the Hawking flux obtained from higher spin anomaly. It corresponds to higher spin moments of Hawking flux. These results have earlier appeared in [18] using anomaly (covariant) cancellation mechanism [13]. Also, recently Sonora and collaborators 
have computed the Hawking fluxes associated with the higher spin currents, using the usual trace anomaly and the properties of $W_{\infty}$ algebra [24, 25].

Here we would present an alternative derivation based on the approach briefly discussed in [2]. An important advantage of this approach is that the computation involved only the expressions for anomalous covariant Ward identities and the covariant boundary conditions. The splitting of space into two regions, which was essential in the approaches [11, 12, 13, 20], is avoided. First, for the sake of completeness, we shall discuss the covariant anomaly approach [2] to compute the fluxes of Hawking radiation associated with the gauge current and stress tensor in detail. Then, it is easy to generalise this analysis to obtain the Hawking fluxes associated with the higher spin currents.

For the right moving modes the expression for covariant gauge anomaly is given by $[8,9]$,

$$
\nabla_{\mu} J^{\mu}=-\frac{e^{2}}{4 \pi \sqrt{-g}} \varepsilon^{\alpha \beta} F_{\alpha \beta} .
$$

Note that the same Ward identity (2.15) was also obtained in the previous section by exploiting structure of the chiral effective action.

For a static background (2.2), equation (3.1) becomes,

$$
\partial_{r}\left(\sqrt{-g} J^{r}\right)=\frac{e^{2}}{2 \pi} \partial_{r} A_{t} .
$$

Solving this equation we get

$$
\sqrt{-g} J^{r}=c_{H}+\frac{e^{2}}{2 \pi}\left[A_{t}(r)-A_{t}\left(r_{h}\right)\right]
$$

Here $c_{H}$ is an integration constant which can be fixed by imposing the covariant boundary condition (2.33) lead to $c_{H}=0$ and hence the expression for the current becomes,

$$
J^{r}=\frac{e^{2}}{2 \pi \sqrt{-g}}\left[A_{t}(r)-A_{t}\left(r_{h}\right)\right]
$$

Note that the Hawking flux is measured at infinity where there is no anomaly. This necessitated a split of space into two distinct regions - one near the horizon and one away from it - and the use of two Ward identities [11, 12, 13, 20]. This is redundant if we observe that the anomaly (3.2) vanishes at the asymptotic infinity. Consequently, in this approach, the flux is directly obtained from the asymptotic infinity limit of (3.4):

$$
\text { Chargeflux }=J^{r}(r \rightarrow \infty)=-\frac{e^{2} A_{t}\left(r_{h}\right)}{2 \pi} .
$$

This reproduces the familiar expression for the charge flux $[1,2,12,13]$.

Next, we consider the expression for the two dimensional covariant gravitational Ward identity $[1,13,19]$ (see also (e.2.16) in the previous section),

$$
\nabla_{\mu} T^{\mu v}=J_{\mu} F^{\mu v}+\frac{\varepsilon^{v \mu}}{96 \pi \sqrt{-g}} \nabla_{\mu} R
$$

and $R$ is given in (2.14). Now by simplifying (3.6) we get, in the static background,

$$
\partial_{r}\left(\sqrt{-g} T_{t}^{r}\right)=\partial_{r} N_{t}^{r}(r)-\frac{e^{2} A_{t}\left(r_{h}\right)}{2 \pi} \partial_{r} A_{t}(r)+\partial_{r}\left(\frac{e^{2} A_{t}^{2}(r)}{4 \pi}\right)
$$


where $N_{t}^{r}(r)$ is given in (2.40). The solution for (3.7) is given by

$$
\sqrt{-g} T_{t}^{r}=b_{H}+\left[N_{t}^{r}(r)-N_{t}^{r}\left(r_{h}\right)\right]+\frac{e^{2} A_{t}^{2}\left(r_{h}\right)}{4 \pi}-\frac{e^{2}}{2 \pi} A_{t}\left(r_{h}\right) A_{t}(r)+\frac{e^{2} A_{t}^{2}(r)}{4 \pi} .
$$

Here $b_{H}$ is an integration constant . Implementing the covariant boundary condition (2.34) yields $b_{H}=0$. Hence (3.8) reads

$$
\sqrt{-g} T_{t}^{r}(r)=\left[N_{t}^{r}(r)-N_{t}^{r}\left(r_{h}\right)\right]+\frac{e^{2}}{4 \pi}\left[A_{t}(r)-A_{t}\left(r_{h}\right)\right]^{2} .
$$

Since the covariant gravitational anomaly (3.6) vanishes asymptotically, we can compute the energy flux as before by taking the asymptotic limit of (3.9)

$$
\begin{aligned}
\text { energyflux }= & T^{r}{ }_{t}(r \rightarrow \infty)=-N_{t}^{r}\left(r_{h}\right)+\frac{e^{2} A_{t}^{2}\left(r_{h}\right)}{4 \pi} \\
& =\frac{1}{192 \pi} f^{\prime}\left(r_{h}\right) g^{\prime}\left(r_{h}\right)+\frac{e^{2} A_{t}^{2}\left(r_{h}\right)}{4 \pi} .
\end{aligned}
$$

This reproduces the expression for the Hawking flux found by using the anomaly cancelling approach of $[1,12,13,20]$.

Now we concentrate our attention on the higher spin currents. Particularly, we shall do our analysis for spin 3 current $J^{\mu}{ }_{v}$. The covariant expression for divergent anomaly for spin 3 current is given by [18],

$$
\begin{aligned}
\nabla_{\mu} J_{v \rho}^{\mu}= & -F_{v \mu} T_{\rho}^{\mu}-F_{\rho \mu} T_{v}^{\mu}-\frac{\nabla_{v}\left(R J_{\rho}\right)}{16}-\frac{\nabla_{\rho}\left(R J_{v}\right)}{16} \\
& +\frac{1}{16} g_{v \rho} \nabla_{\mu}\left(R J^{\mu}\right)+\frac{1}{24 \sqrt{-g}}\left[\varepsilon_{v \sigma} \nabla^{\sigma} \nabla_{\mu} F_{\rho}^{\mu}\right. \\
& \left.+\varepsilon_{\rho \sigma} \nabla^{\sigma} \nabla_{\mu} F_{v}^{\mu}-g_{v \rho} \varepsilon_{\alpha \sigma} \nabla^{\sigma} \nabla_{\mu} F^{\mu \alpha}\right]
\end{aligned}
$$

here $T^{\mu}{ }_{v}$ and $J^{\mu}$ are covariant EM tensor and U(1) current respectively. In the above expression term in the square bracket represents the anomalous (quantum) correction to the classical result. This piece is the spin 3 generalization of the divergence anomalies in the electric and gravitational backgrounds. Note that the expectation value of the current depends only on $r$ in the static black hole background taken here (2.2), hence, the relevant $v=\rho=t$ component of (3.11) becomes,

$$
\begin{aligned}
\partial_{r}\left(\sqrt{-g} J_{t t}^{r}\right)= & -2 F_{t r} T^{r}{ }_{t}-\frac{1}{8} \nabla_{t}\left(R J_{t}\right)+\frac{1}{16} g_{t t} \nabla_{\mu}\left(R J^{\mu}\right) \\
& +\frac{1}{24}\left[-2 \nabla^{r} \nabla_{\mu} F_{t}^{\mu}{ }_{t}-g_{t t} \varepsilon_{\alpha \sigma} \nabla^{\sigma} \nabla_{\mu} F^{\mu \alpha}\right] .
\end{aligned}
$$

The solution for above equation is given by

$$
\begin{aligned}
\sqrt{-g} J_{t t}^{r}= & D_{H}+\int_{r_{h}}^{r} d r \partial_{r}\left[\frac{f^{\prime}\left(r_{h}\right) h^{\prime}\left(r_{h}\right)}{96 \pi} A_{t}(r)-\frac{e^{2} A_{t}\left(r_{h}\right)}{2 \pi} A_{t}^{2}(r)-\frac{e^{2} A_{t}\left(r_{h}\right)}{32 \pi}\left(h f^{\prime \prime}+f^{\prime} h^{\prime}-\frac{2 f^{\prime 2} h}{f}\right)\right. \\
& \left.+\frac{A_{t}^{3}(r)}{6 \pi}-\frac{h f \partial_{r}^{2} A_{t}(r)}{96 \pi}+\frac{h f^{\prime} \partial_{r} A_{t}(r)}{48 \pi}+\frac{\left(h f^{\prime \prime}+f^{\prime} h^{\prime}-\frac{2 f^{\prime 2} h}{f}\right) A_{t}(r)}{32 \pi}\right] .
\end{aligned}
$$


where $D_{H}$ is an integration constant and its value is determined by imposing the covariant boundary condition, $J_{t t}^{r}\left(r=r_{h}\right)=0$, this yield $D_{H}=0$. Now, the anomalous part in (3.11) vanish in the asymptotic infinity limit. Hence the Hawking flux for spin 3 current is just given by $J^{r}{ }_{t t}(r \rightarrow \infty)$,

$$
J_{t t}^{r}(r \rightarrow \infty)=-\frac{f^{\prime}\left(r_{h}\right) h^{\prime}\left(r_{h}\right)}{96 \pi}-\frac{A_{t}^{3}\left(r_{h}\right)}{6 \pi} .
$$

This result coincide with appropriate ( $\mathrm{n}=2)$ moment of the Hawking flux [18].

\section{Conclusions}

In this paper we discussed in detail the effective action approach [1] and the covariant anomaly method [2] to compute the Hawking flux from generic spherically symmetric black hole. First, the effective action approach was discussed. As stated in [11,12], if we neglect, classically, the contribution from ingoing modes, then the near horizon effective theory becomes chiral. Consequently, this theory can be represented by the chiral effective action [1]. The covariant expressions for current $J^{\mu}$ and stress tensor $T^{\mu v}$ were then obtained from this chiral effective action, modified by the local counterterm. Expectedly, current and stress tensor satisfy the covariant anomalous Ward identities. Arbitrary constants appearing in the expressions for $J^{r}$ and $T^{r}{ }_{t}$ were fixed by implementing the covariant boundary conditions [13]. Then by noting the fact that covariant gauge as well as gravitational anomalies vanish in the asymptotic infinity limit, the Hawking charge and energy flux were computed by appropriately taking the asymptotic limit of the covariant current and the covariant stress tensor.

Further, we apply the covariant anomaly method [2] to derive the Hawking radiation associated with the higher spin currents. Contrary to earlier approaches [11, 12, 13, 20], this method uses only the covariant gauge and gravitational Ward identities defined near the horizon. Another advantage is that unlike the anomaly (covariant/consistent) cancellation mechanism, splitting of space into two regions - near to and away from the event horizon - using discontinuous step functions, was avoided.

A reason in favor of working with the covariant anomalies is the fact that their functional forms are unique, being governed solely by gauge/diffeomorphism transformations. This is not valid for consistent anomalies. This fact becomes crucial when we discuss the higher spin fluxes. The point is that for higher spin currents only the covariant expressions of anomalies were known [18]. Here we performed the computations for spin 3 current. The fluxes of Hawking radiation were obtained by solving the covariant Ward identity near the horizon. In this case also, in the asymptotic infinity limit, the covariant anomaly vanishes. The Hawking radiation corresponding to spin 3 current was the obtained by taking the asymptotic limit of covariant spin 3 current. Our results matches exactly with the earlier finding [18] based on the anomaly cancellation approach.

Acknowledgments : I thank Prof. Rabin Banerjee for useful suggestions. Also, I would like thank Prof. L. Bonora and Prof. S. Pallua for warm hospitality during the workshop on "Black holes in General Relativity and String Theory" held at Veli Losinj, Croatia. 


\section{References}

[1] R. Banerjee, S. Kulkarni, Hawking Radiation, effective actions and covariant boundary condition, Phys. Lett.B 659, 827 [arXiv:0709.3916 [hep-th]] (2008).

[2] R. Banerjee, Covariant Anomalies, Horizons and Hawking Radiation, [arXiv: 0807.4637 [hep-th] ] (To appear in the special issue of IJMP D) (2008).

[3] J. Bekenstein, Generalized second law of thermodynamics in black hole physics, Phys. Rev. D 93292 (1974).

[4] S. Hawking, Particle Creation by Black Holes, Commun. Math. Phys. 43199 (1975).

[5] G. Gibbons, S. Hawking, Action Integrals and Partition Functions in Quantum Gravity, Phys. Rev. D 152752 (1977).

[6] M. Parikh, F. Wilczek, Hawking radiation as tunneling, Phys. Rev. Lett. 85, 5042 [hep-th/9907001] (2000).

[7] K. Srinivasan, T. Padmanabhan, Particle production and complex path analysis, Phys. Rev. D 60 024007 [gr-qc/9812028] (1999).

[8] R. Bertlmann, Anomalies In Quantum Field Theory, Oxford Sciences, Oxford, 2000.

[9] K. Fujikawa and H. Suzuki, Path Integrals and Quantum Anomalies, Oxford Sciences, Oxford, 2004.

[10] S. Christensen and S. Fulling, Trace Anomalies and the Hawking Effect, Phys. Rev. D 152088 (1977).

[11] S. P. Robinson and F. Wilczek, A Relationship between Hawking radiation and gravitational anomalies, Phys. Rev. Lett. 95011303 [gr-qc/ 0502074 ] (2005).

[12] S. Iso, H. Umetsu and F.Wilczek, Hawking radiation from charged black holes via gauge and gravitational anomalies, Phys. Rev. Lett. 96151302 [hep-th/0602146] (2006).

[13] R. Banerjee, S. Kulkarni, Hawking radiation and covariant anomalies, Phys. Rev. D 77024018 [arXiv:0707.2449 [hep-th]](2008).

[14] H. Banerjee, R. Banerjee and P. Mitra, Covariant And Consistent Anomalies In Even Dimensional Chiral Gauge Theories, Z. Phys. C 32445 (1986).

[15] W. A. Bardeen, B. Zumino, Consistent and Covariant Anomalies in Gauge and Gravitational Theories, Nucl. Phys. B 244421 (1984).

[16] R. Bertlmann and E. Kohlprath, Two-dimensional gravitational anomalies, Schwinger terms and dispersion relations, Ann. Phys. (N.Y.) 288137 [hep-th/ 0011067 (2001).

[17] L. Alvarez-Gaume and E. Witten, Gravitational Anomalies, Nucl. Phys. B 234269 (1984).

[18] S. Iso, T. Morita and H. Umetsu, Hawking Radiation via Higher-spin Gauge Anomalies, Phys. Rev. D 77045007 [arXiv:0710.0456 [hep-th]].

[19] H. Leutwyler, Gravitational Anomalies: A Soluble Two-Dimensional Model,Phys. Lett. B 153, 65 (1985).

[20] S. Iso, H. Umetsu and F. Wilczek, Anomalies, Hawking radiations and regularity in rotating black holes, Phys. Rev. D 74, 044017 [hep-th/ 0606018$]$ (2006).

[21] R. Balbinot, A. Fabbri, I. Shapiro, Vacuum polarization in Schwarzschild space-time by anomaly induced effective actions, Nucl. Phys.B 559, 301 [hep-th/9904162] (1999). 
[22] R. Banerjee, S. Kulkarni, Hawking Radiation, Covariant Boundary Conditions and Vacuum States, [arXiv:0810.5683 [hep-th]].

[23] E. C. Vagenas, S. Das, Gravitational anomalies, Hawking radiation, and spherically symmetric black holes, JHEP 0610, 025 [hep-th/ 0606077 ] (2006).

[24] L. Bonora, M. Cvitan, Hawking radiation, W-infinity algebra and trace anomalies, JHEP $\mathbf{0 8 0 5}, 071$ [arXiv:0804.0198 [hep-th]](2008).

[25] L. Bonora, M. Cvitan, S. Pallua, I. Smolic, Hawking Fluxes, W(infinity) Algebra and Anomalies, [arXiv:0808.2360 [hep-th]] (2008). 